\title{
Comparison of P53 Mutation Status, Primary Cytoreductive Surgical Outcomes, and Overall Survival in Patients with Ovarian Cancer
}

\author{
D Engle, ${ }^{1}$ H Fornalik, ${ }^{1} \mathrm{~T}$ Neff, ${ }^{1}$ A Button, ${ }^{2} \mathrm{~K}$ Collins, ${ }^{3}$ A Ahmed,${ }^{1,4} \mathrm{~K}$ DeGeest, ${ }^{1,4} \mathrm{D}$ \\ Bender, ${ }^{1,4} \mathrm{~S}$ Lutgendorf, ${ }^{3} \mathrm{M}$ Goodheart ${ }^{1,4}$
}

Objective: We examined both p53 mutation status and primary surgical cytoreduction status in ovarian cancer patients to define possible characteristics associated with improved survival.

Methods: 270 ovarian cancer samples were obtained from a large divisional tumor bank. cDNA was extracted from stored tumor tissue, and the entire open reading frame (exon 2-11) was amplified and sequenced in both directions. Patients were then divided into 2 cohorts based on p53 mutational status. Surgical, clinical and survival data was then obtained.

Results: Of the 270 identified patients, 258 were evaluable for mutation status and clinical correlation. 160 (62\%) patients were found to have a p53 mutation. Poorly differentiated tumors were more frequently associated with p53 mutations $(80 \%$ vs. $67 \%$; $p=0.0015)$. No statistically significant difference between stage, histology, or overall survival was noted between the 2 groups.

Optimal cytoreduction, $\mathrm{n}=162 \quad(64 \%)$, was not different between mutated $(61 \%)$ or non-mutated groups $(65 \%$; $\mathrm{p}=0.59$ ). Optimal cytoreduction was however, associated with a statistically significant better overall survival among both the p53 mutated $(p=0.0007)$ and non-mutated patients $(p=<0.0001)$. In the p53 mutation group the median survival for both the optimal and suboptimal patients is approximately 4 years. While in the non-mutated group the median survival is 5 years for the optimally cytoreduced vs. 1 year for the suboptimal.

${ }^{1}$ Department of Obstetrics \& Gynecology, ${ }^{2}$ Department of Biostatistics, ${ }^{3}$ Department of Psychology, ${ }^{4}$ Holden Comprehensive Cancer Center, The University of lowa, lowa City, lowa 52242

Corresponding author: Michael Goodheart, Department of Obstetrics and Gynecology, University of lowa, 3234 MERF, 200 Hawkins Drive, lowa City, IA, 52242. Telephone(319) 335-2015 michael-goodheart@uiowa.edu 
WESTERN ASSOCIATION OF GYNECOLOGIC ONCOLOGISTS (WAGO)

WAGO Annual Meeting

June 16-18, 2011

Stein Eriksen Lodge

Park City, Utah

Multivariate analysis showed p53 survival that favors the p53 mutation mutation was not predictive of survival for optimally cytoreduced patients $(\mathrm{HR}=1.315 ; 95 \%$, Cl 0.851-2.031). The risk of death decreased in suboptimally cytoreduced patients with a p53 mutation (HR=0.553; 95\%, Cl 0.3430.890). Additionally, for those patients who are suboptimally cytoreduced there is a statistically significant difference in group (p-0.015).

Conclusions: Optimal surgical cytoreduction continues to be an important prognostic variable in patients with ovarian cancer independent of p53 mutational status. Among patients with suboptimal cytoreduction a p53 mutation may offer a survival advantage.

P53 mutation, cytoreductive surgery and survival 